\section{Correspondence}

https://doi.org/10.11646/zootaxa.5016.3.10

http://zoobank.org/urn:lsid:zoobank.org:pub:354D1F85-F40C-41EF-84BA-645B5C3756A7

\title{
Dissecting the tree of life: the prospect of open-access digital resources in morphology, anatomy and taxonomy in training the next generation of zoologists
}

\author{
KATHARINA RUTHSATZ ${ }^{1, *}$, MARK D. SCHERZ ${ }^{2}$ \& MIGUEL VENCES ${ }^{3}$ \\ ${ }^{1}$ Department of Evolutionary Biology, Zoological Institute, Technische Universität Braunschweig, Mendelssohnstraße 4, 38106 \\ Braunschweig, Germany \\ =" katharinaruthsatz@gmail.com; @ https://orcid.org/0000-0002-3273-2826 \\ ${ }^{2}$ Faculty of Mathematics and Natural Sciences, Institute for Biochemistry and Biology, University of Potsdam, Potsdam, Germany. \\ "= mark.scherz@gmail.com; (1) https://orcid.org/0000-0002-4613-7761 \\ ${ }^{3}$ Department of Evolutionary Biology, Zoological Institute, Technische Universität Braunschweig, Mendelssohnstraße 4, 38106 \\ Braunschweig, Germany. \\ झ"m.vences@tu-braunschweig.de; 애ttps://orcid.org/0000-0003-0747-0817 \\ * corresponding author
}

While a variety of new research fields have emerged in biology over the decades, basic disciplines such as anatomy and morphology remain the foundation of biological education, and the future of taxonomic research heavily depends on recruiting scientists familiar with detailed analysis of phenotypic characters. A comprehensive understanding of the basic descriptive disciplines of biology—which address Mayr's (1998) "What?" questions in biology—is also of key importance for students and researchers to understand "How?" questions related to physiological, biochemical and developmental processes in an organismal context, genome-phenotype interactions, or the ecological relevance and services of organismal diversity. However, especially with the rise of ever-more efficient "-omics" techniques, the number of courses teaching the traditional bases of biology has severely declined in favor of integrating new disciplines such as genetics, molecular cell biology, biochemistry, or bioinformatics into the university curriculum.

The rise of a new generation of biology students who are especially attracted to digitalization (Farnan et al. 2008; Barry et al. 2016) and to the use of social media offers new opportunities for scientific education at university level. Using guided online courses in addition to classroom teaching might in part compensate for the reduced capacities of universities in basic discipline training. This is particularly true for zoology, since dissection courses are time-consuming and require a substantial amount of preparation and follow-up learning. Digital resources such as dissection videos might be used as a tool to compensate for time constraints in zoological education. Furthermore, these videos offer a comfortable possibility for zoologists in teaching to prepare for the hands-on anatomy lab courses, and to refresh this knowledge whenever it is needed.

Surprisingly, despite an enormous boost of digital teaching due to Covid-19-related restrictions in 2020 (Skulmowski \& Rey 2020), the amount of open-access online teaching resources in zoology is relatively scarce beyond rather generalized high-school-level materials. As an example of the kind of materials that are, in our opinion, badly needed, we here present an entire season of 14 guided video courses in animal anatomy and morphology that were prepared for teaching a general zoology lab for undergraduates at Braunschweig University of Technology and are available (in German language) from the University's YouTube channel (https://www.youtube.com/playlist?list=PLLxinzTP5QP2Qzyzm47dVAt2uX5k I59FV). These videos are also being made available in English and German via a dedicated YouTube channel called Anatomy Insights (https://www.youtube.com/channel/UCsDnwZXBw1yqdixNvj-zjmA). They cover all major taxa which are traditionally included in zoology curricula at university level (e.g., protists, cnidarians, platyhelminths, rotifers, nematodes, molluscs, hexapods, crustaceans, echinoderms, tunicates, cephalochordates, teleosts, amphibians, squamates, birds, and mammals). The videos give a brief overview of the taxonomy, biology, and ecology of the respective taxa and provide a step-by-step/real-time manual for dissection. Because many of the taxa vary significantly in bauplan, most videos include not just one subject, but a broad phylogenetic sample for each group. For instance, our squamate video includes a gekkonid gecko (Phelsuma standingi, with male secondary sexual characters highlighted in a second species, $P$. quadriocellata, and gross anatomical differences between Gekkonidae and Diplodactylidae highlighted by comparison to Rhacodactylus auriculatus), an agamid lizard (Pogona vitticeps), a chameleon (Chamaeleo calyptratus, with hemipenis preparation method demonstrated on Trioceros hoehnelii), and a snake (Pantherophis guttatus). 
We emphasize that the primary benefit of these dissection videos comes from using them as a supplemental teaching aid especially for preparation, follow-up learning, and knowledge refreshment for both students and lecturers. In particular, we recommend using these videos as a pre-dissection introduction because they set the students up for learning much more deeply about the actual hands-on dissection. Animal dissection has often been criticized for being ethically contentious (Bishop \& Nolan 2001; Oakley 2012). We are convinced that hands-on examination of biological materials, including dissection of animals, should remain an integral component of biology curricula, and that creative solutions to minimize the number of animals sacrificed for this purpose exist. For instance, if all necessary veterinary and nature conservation permits are in place, fresh roadkills of amphibians, reptiles or mammals can be used for dissection; students can take turns to dissect a specimen; or, if adequate hygiene protocols are followed, parts of dissected animals such as fishes might even be set aside and used for human consumption. Obviously, sacrificing threatened species for teaching should be avoided at all costs. Depending on the curriculum, dissection of some animal groups might also be skipped, and in this context our videos can help to replace specific teaching units and thereby reduce the usage of animals. Furthermore, the videos provide a no-cost alternative to the often expensive practical dissection. Since they can be re-used year after year, they thus have a considerably a lower cost and environmental and ethical footprint than traditional university courses.

Dissection videos as a complement or partial alternative to hands-on lab courses have been successfully proven to support students in multiple scientific disciplines where anatomical knowledge is important, such as medicine (Granger \& Calleson 2007; Topping 2014), neurobiology (Williams et al. 2019), and dental science (Mukhopadhyay et al. 2014; Iwanaga et al. 2020). However, only limited research has been published on the use of platforms such as YouTube as a tool for education in a university setting in general (rev. in Jaffar 2012), and in zoology education in particular. In the future, such digital platforms might increasingly provide a supplementary e-resource for biological education. Being free to access, and almost globally available, they can also serve to democratize access to anatomical education opportunities. As the acceptance of e-learning tools was slightly declining before the current Covid-19 outbreak, it is to be assumed that the demand for digital offers will rather increase (Zawacki-Richter 2020). Consequently, digital resources like ours might contribute to university remote teaching on the long-term scale. However, we once more emphasize that there is no substitute for practical anatomical experience, and dissection videos must not completely replace hands-on dissection in anatomy courses at university level.

Our future work will add further such dissection videos with a broadened taxonomic scope, including taxa that are particularly worth presenting and that usually do not appear in zoological curricula at university level. While dissecting through the tree of life, we will increasingly introduce modern techniques such as micro-CT scanning and high-resolution imaging to foster the enthusiasm of students for the study of animal body plans. Eventually, besides videos, the elaboration of more interactive teaching resources could be a suitable tool to increase the visibility of our discipline, including basic procedures of taxonomy. For instance, our reptile video already includes a section on the preparation of hemipenes, the anatomy of which is important for taxonomic purposes, but the preparation of which is not - to our knowledgedemonstrated in any other existing YouTube video, and cannot easily be communicated in text form. This might also be particularly useful to the many amateur taxonomists who are often self-taught, and thus can deepen their knowledge without the need of attending university courses.

Strikingly, so far there seem to be no dedicated open-access platforms allowing researchers to publish such selfelaborated teaching resources under a peer-review process, in order to get credit for their effort. We are convinced that high-quality and open-access digital teaching resources, including introductions to state-of-the-art methods, can substantially contribute to attract (student) zoologists into comparative and functional anatomy, and hopefully also taxonomy, as modern scientific disciplines.

\section{Acknowledgements.}

We are grateful to various colleagues for discussion, materials and assistance, in particular Otto Larink, Ilka Sötje, and Matthias Overmann, as well as to Salvador Carranza and an anonymous reviewer for constructive comments on the initial manuscript.

\section{References}

Bishop, L.J. \& Nolen, A.L. (2001) Animals in research and education: Ethical issues. Kennedy Institute of Ethics Journal, 11, 91-112.

https://doi.org/10.1353/ken.2001.0006 
Farnan, J.M., Paro, J.A., Higa, J., Edelson, J. \& Arora, V.M. (2008) The YouTube generation: implications for medical professionalism. Perspectives in Biology and Medicine, 51, 517-524.

https://doi.org/10.1353/pbm.0.0048

Granger, N.A. \& Calleson, D. (2007) The impact of alternating dissection on student performance in a medical anatomy course: Are dissection videos an effective substitute for actual dissection?. Clinical Anatomy: The Official Journal of the American Association of Clinical Anatomists and the British Association of Clinical Anatomists, 20, 315-321.

https://doi.org/10.1002/ca.20359

Iwanaga, J., Tanaka, T., Ohyama, H. \& Tubbs, R.S. (2020) Online remote interactive lecture for postgraduate dental education in clinical anatomy. Journal of Dental Education, 85, 985-987. https://doi.org/10.1002/jdd.12335

Jaffar, A.A. (2012) YouTube: An emerging tool in anatomy education. Anatomical Sciences Education, 5, $158-164$. https://doi.org/10.1002/ase.1268

Mayr, E. (1998) This is Biology: The Science of the Living World. Harvard University Press, Cambridge, Massachusetts, xix + $323 \mathrm{pp}$.

Mukhopadhyay, S., Kruger, E. \& Tennant, M. (2014) YouTube: a new way of supplementing traditional methods in dental education. Journal of Dental Education, 78, 1568-1571. https://doi.org/10.1002/j.0022-0337.2014.78.11.tb05833.x

Oakley, J. (2012) Science teachers and the dissection debate: perspectives on animal dissection and alternatives. International Journal of Environmental and Science Education, 7, 253-267.

Skulmowski, A. \& Rey, G.D. (2020) COVID-19 as an accelerator for digitalization at a German university: Establishing hybrid campuses in times of crisis. Human Behavior and Emerging Technologies, 2, 212-216. https://doi.org/10.1002/hbe2.201

Topping, D.B. (2014) Gross anatomy videos: Student satisfaction, usage, and effect on student performance in a condensed curriculum. Anatomical Sciences Education, 7, 273-279. https://doi.org/10.1002/ase.1405

Williams, S.R., Thompson, K.L., Notebaert, A.J. \& Sinning, A.R. (2019) Prosection or dissection: Which is best for teaching the anatomy of the hand and foot?. Anatomical Sciences Education, 12, 173-180.

https://doi.org/10.1002/ase.1808 